
cylindrical structure

\title{
Modelling of water wave interaction with multiple cylinders of arbitrary shape
}

\author{
Hao Song ${ }^{1}$, Longbin $\mathrm{Tao}^{2}{ }^{*}$ and Subrata Chakrabarti ${ }^{3}$ \\ ${ }^{a}$ Griffith School of Engineering, Griffith University, Gold Coast, QLD4222, Australia \\ ${ }^{\mathrm{b}}$ School of Marine Science and Technology, Newcastle University, NE1 7RU, UK \\ ${ }^{\mathrm{c}}$ Offshore Structure Analysis, Inc.,Plainfield, IL60544-7096, USA
}

\begin{abstract}
This paper describes the development of an efficient numerical model, namely scaled boundary finite-element method (SBFEM) for linear waves interaction with cylindrical structures of arbitrary shapes. The two-dimensional Helmholtz equation is firstly weakened in the circumferential direction, so that the governing partial differential equation is transformed to an ordinary matrix differential equation in radial direction, and is solved fully analytically. As a key element, a virtual porous circular cylinder surrounding the cylindrical structures is introduced so that the entire computational domain is partitioned along the virtual cylinder into an unbounded and several bounded sub-domains with common interfaces. The principle innovation is that, the present SBFEM model chooses Hankel function as a base solution for the unbounded sub-domain, while a power series is used for the internal bounded subdomains. The approach discretises only the common interfaces of the sub-domains with surface finite-elements, and fewer elements are required to obtain very accurate results. Numerical simulations show that the new SBFEM model offers a considerable improvement by far in its numerical performance, as well as in the range of physical phenomena that is capable of simulating. The wave forces and run-ups are presented for a single and multiple cylindrical structures of different cross sectional shapes. Influences of the incident wave parameters and structural configurations on the hydrodynamics are examined.
\end{abstract}

Key words: scaled boundary finite-element method, wave diffraction, unbounded domain, 
* Corresponding author. Tel.: +44 (0)191 222 6670; fax: +44 (0)191 2225491. Email address: L. Tao@ncl . ac.uk (Longbin $\left.\mathrm{Tao}^{2}\right)$. 
dent waves especially in three-dimensional problems, the whole computation work is enormous. BEM, on the other hand, has the inherent advantage for wave-structure interaction in unbounded domain with the property of reducing the spatial dimension by one. However, fundamental solutions are required and singular integrals exist. Futhermore, it may suffer from the problems caused by irregular frequencies and sharp corner.

Recently, the scaled boundary finite-element method (SBFEM), originally developed to solve soil-structure interaction problems (e.g., [9]), has been successfully applied to water wave diffraction, in which the radiation condition at infinity is required to be satisfied by the scattered waves. Tao et al. [10] applied the SBFEM to solve short-crested waves interaction with a circular cylinder. Instead of using an algebraic series, Tao et al. [10] chose Hankel function to solve the Helmholtz equation in the unbounded domain. The radial differential equation is solved fully analytically in all frequency ranges. Without relying on any other numerical schemes, the semi-analytical model for the wave diffraction by a circular cylinder is shown to reproduce the analytical solution for all the physical properties including wave run-ups, effective inertia and drag coefficients, and total force very accurately and at very low computational cost.

Most of the approximate theories for simple structure geometries, as well as the numerical solutions for the two-dimensional structures of circular cross section provide an important step in understanding the effects of wave diffraction on large bodies. The solutions have a wide range of applications, but are limited by the special geometry and are generally not applicable to large offshore structures of general geometry. Hence, it becomes necessary to take up the case of cylindrical structures of arbitrary cross section in order to deal with the variety and complexity of design configurations encountered in modern offshore structures. 
In this paper, the SBFEM model is further extended to solve water waves interaction

with: 1) a single cylindrical structure of arbitrary shape; 2) multiple structures system. The present approach applied domain decomposition technique by introducing a porous circular cylinder surrounding a single or multiple cylinders of arbitrary cross section. The fluid domain is therefore divided into an unbounded sub-domain and several bounded sub-domains. For the outer unbounded sub-domain, a semianalytical solution is obtained by employing a base solution in terms of the Hankel function of the first kind which satisfies the radiation boundary condition at infinity, while for the bounded sub-domains, the semi-analytical solutions are given by matrix power series. Detailed numerical results on wave forces and run-ups over broad range of incident wave parameters as well as structure configurations are presented.

\section{Mathematical model and numerical implementation}

\subsection{Boundary value problem}

Consider a monochromatic wave train propagating at an angle $\theta$ with positive $x$ axis. A structure system consisting of several vertical cylinders extends from the sea bottom to above the free surface of the ocean along $z$ axis (see Fig. 1).

Tao et al. [10] showed that the solution process can be significantly simplified by choosing the Hankel function as a base function for wave diffraction by a circular cylinder. However, it is no longer valid for a cylinder with arbitrary cross section. In order to preserve the accuracy and efficiency of the SBFEM model and overcome the convergence problem associated with the algebraic series base function, an artificial porous circular cylinder $\left(\Gamma_{c}\right)$ enclosing the structure system consisting of several vertical cylinders is introduced. The origin is placed at the centre of the ex- 

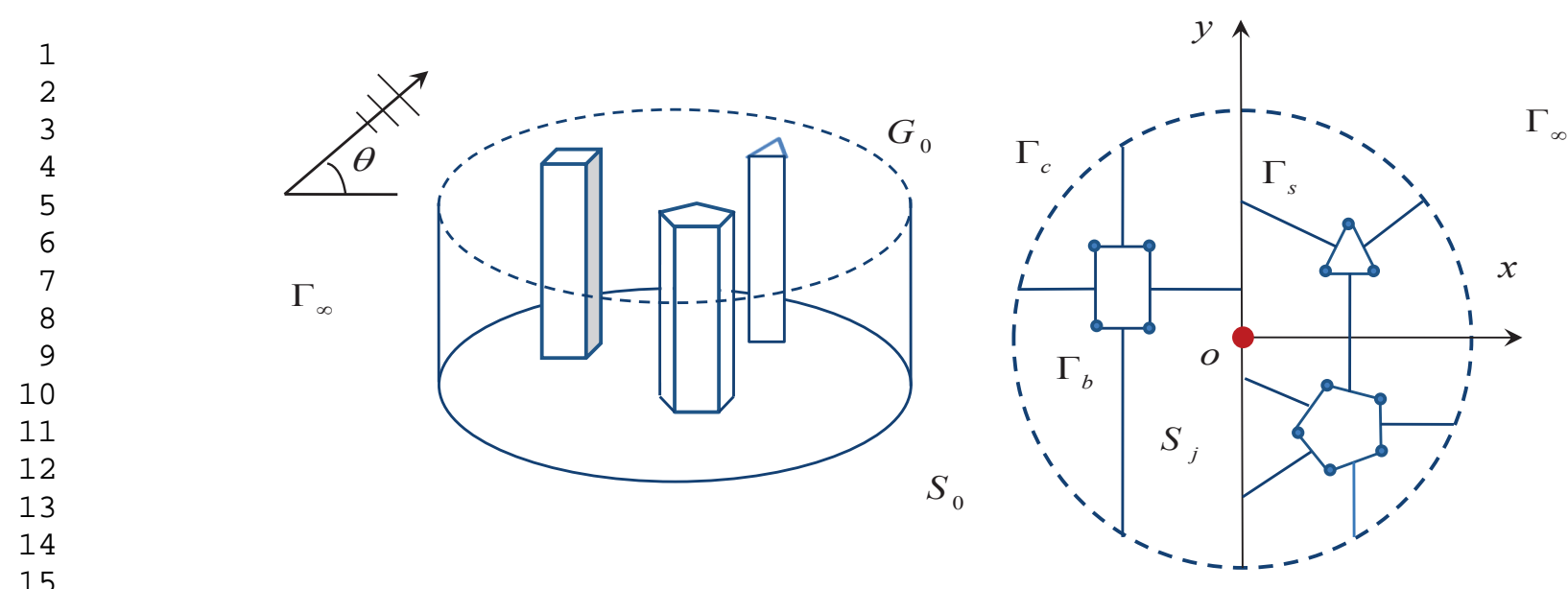

Fig. 1. A sketch of the water wave diffraction by multiple bodies.

terior circular cylinder on the mean water surface (Fig. 1). The whole fluid region is then divided into two regions, the interior bounded region and the unbounded region outside of the cylinder $S_{0}$. The interior region is further divided into $q$ subdomains, $S_{1}, S_{2}, \ldots, S_{q}$. The following notation have been used in the paper: $\Phi_{j}=$ total velocity potential in $j$ th sub-domain, $\Phi_{0}^{I}=$ velocity potential of incident wave in $S_{0}, \Phi_{0}^{S}=$ velocity potential of scattered wave in $S_{0}, k=$ total wave number, $k_{x}=$ wave number in $x$ direction, $k_{y}=$ wave number in $y$ direction, $\omega=$ wave frequency, $h=$ water depth, $A=$ amplitude of incident wave, $a=$ the characteristic length of the interior cylinders, $c=$ the radius of the porous circular cylinder, $t=$ time, $\rho=$ mass density of water, and $g=$ gravitational acceleration. The subscripts $j(j=0,1,2, \ldots, q)$ denote the physical parameters in the solution sub-domain $S_{j}$.

\footnotetext{
The velocity potentials can be decomposed by separating the vertical variable $z$ and the time $t$ from each component as
} 
$\Phi_{j}(x, y, z, t)=\phi_{j}(x, y) Z(z) e^{-i \omega t} \quad$ in $\quad S_{j}$

$\Phi_{0}^{I}(x, y, z, t)=\phi_{0}^{I}(x, y) Z(z) e^{-i \omega t} \quad$ in $\quad S_{0}$,

$\Phi_{0}^{S}(x, y, z, t)=\phi_{0}^{S}(x, y) Z(z) e^{-i \omega t} \quad$ in $\quad S_{0}$,

where

$$
Z(z)=\frac{\cosh k(z+h)}{\cosh k h}
$$

113

leading to the seabed boundary condition being satisfied. The diffraction problem in $S_{0}$ is then governed by Helmholtz equation with the boundary condition at the porous interface $\Gamma_{c}$, and the radiation condition at infinity:

$$
\begin{array}{r}
\phi_{0, n}^{S}+\phi_{0, n}^{I}=-\phi_{\operatorname{adj}, n}=-i G_{0} k\left(\phi_{\operatorname{adj}}-\phi_{0}^{S}-\phi_{0}^{I}\right) \quad \text { on } \quad \Gamma_{c}, \\
\lim _{k r \rightarrow \infty}(k r)^{1 / 2}\left(\phi_{0, r}^{S}-i k \phi_{0}^{S}\right)=0 \quad \text { on } \quad \Gamma_{\infty},
\end{array}
$$

where $G_{0}$ is a measure of the porous effect [11] and $G_{0}=0, \infty$ represent a solid wall and a transparent boundary respectively, $r$ is the radial axis, $i=\sqrt{-1}$ is the imaginary unit, $n$ denotes the normal to the boundary, "adj" in the subscript denotes the physical quantities in the adjacent sub-domain, and comma in the subscript designates the partial derivative with respect to the following variable.

The function $\phi_{j}(x, y)(j=1,2, \cdots, q)$ in the interior region is governed by the Helmholtz equation with the boundary conditions at the interface of the sub-domains $\Gamma_{s}$ and $\Gamma_{c}$, and body boundary $\Gamma_{b}$ : 


$$
\begin{aligned}
& \nabla^{2} \phi_{j}+k^{2} \phi_{j}=0 \quad \text { in } \quad S_{j}, \\
& \phi_{j}=\phi_{\mathrm{adj}} \quad \text { on } \Gamma_{s}, \\
& \phi_{j, n}=-\phi_{\operatorname{adj}, n} \quad \text { on } \quad \Gamma_{s}, \\
& \phi_{j, n}=-\phi_{0, n}^{S}-\phi_{0, n}^{I}=i G_{0} k\left(\phi_{j}-\phi_{0}^{S}-\phi_{0}^{I}\right) \quad \text { on } \quad \Gamma_{c} \text {, } \\
& \phi_{j, n}=0 \quad \text { on } \quad \Gamma_{b} .
\end{aligned}
$$

According to Mei [12], the linear incident plane wave can be expressed by the real part of

$$
\Phi_{I}=-\frac{i g A}{\omega} Z(z) e^{i\left(k_{x} x+k_{y} y-\omega t\right)}
$$

and the relationship of total velocity potential, scattered wave, and incident wave velocity potentials are

$$
\Phi_{0}=\Phi_{0}^{I}+\Phi_{0}^{S}, \quad \phi_{0}=\phi_{0}^{I}+\phi_{0}^{S}
$$

Eqs (5)-(12) constitute two sets of the governing equation and boundary conditions for the diffraction of plane waves by a cylindrical structure system with a porous surrounding circular cylinder, corresponding to boundary value problems in several bounded sub-domains and an unbounded sub-domain respectively. The boundary condition on the porous interface is eliminated by matching the unbounded subdomain solution and bounded sub-domain solutions on $\Gamma_{c}$, corresponding to $G_{0}=$ $\infty$. After obtaining $\Phi_{j}$ by solving the above boundary-value problems, the velocity, free surface elevation and the dynamic pressure can be calculated respectively from 


$$
\begin{aligned}
\mathbf{v}_{j} & =\nabla \Phi_{j}, \\
\eta_{j} & =\frac{i \omega}{g} \phi_{j}, \\
p_{j} & =-\rho \Phi_{j, t} .
\end{aligned}
$$

\subsection{Scaled boundary finite-element transformation}

$$
\phi_{, n}=\bar{v}_{n}, \quad \text { on } \quad \Gamma_{v}
$$

where the overbar denotes a prescribed value.

The finite-element method requires the weighted residuals of the governing equation to be zero. Hence Eqs. (5), (8) and (18) are multiplied by a weighting function $w$ and integrated over the flow domain and the boundary. Performing integration by parts, the resulting equation becomes

$$
\int_{\Omega} \nabla^{T} w \nabla \phi d \Omega-\int_{\Omega} w k^{2} \phi d \Omega-\oint_{\Gamma} w \bar{v}_{n} d \Gamma=0
$$

SBFEM defines the domain $\Omega$ by scaling a single piecewise-smooth curve $S$ relative to a scaling centre $\left(x_{0}, y_{0}\right)$, which is chosen at the centre of the porous cylinder in this case (see Fig. 2). The circumferential coordinate $s$ is anticlockwise along the curve $S$ and the normalised radial coordinate $\xi$ is a scaling factor, defined as 1 at curve $S$ and 0 at the scaling centre. The whole solution domain $\Omega$ is in the range 


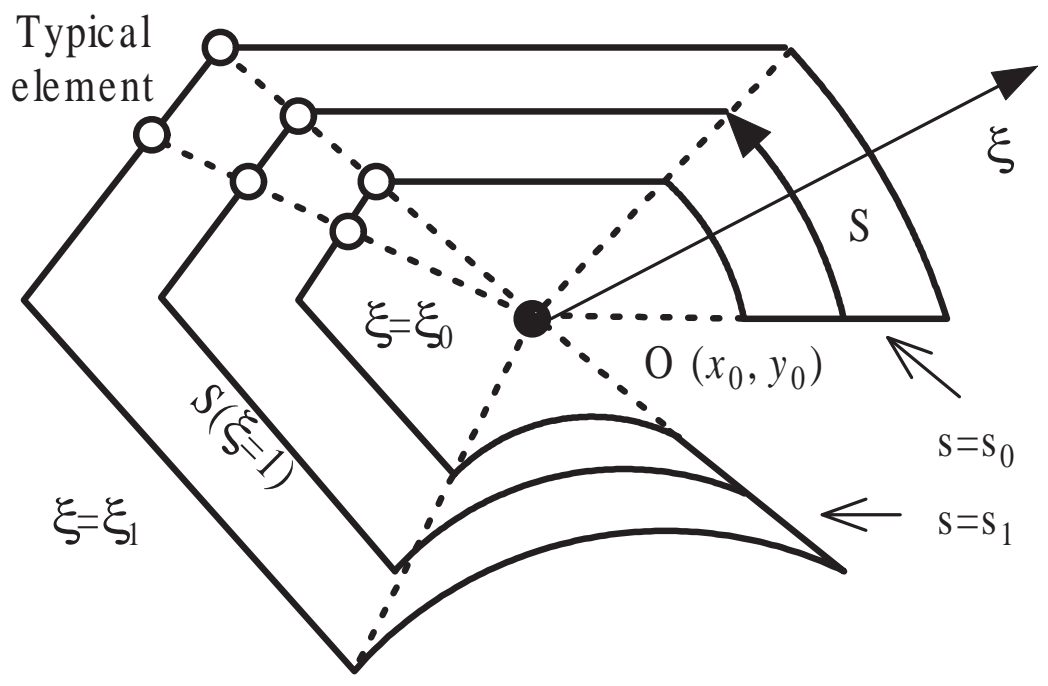

Fig. 2. The coordinate definition of SBFEM

$$
x=x_{0}+\xi x_{s}(s), \quad y=y_{0}+\xi y_{s}(s) .
$$

By employing SBFEM, an approximate solution of $\phi$ is sought as

$$
\phi_{A}(\xi, s)=\mathbf{N}(s) \mathbf{a}(\xi)
$$

where $\mathbf{N}(s)$ is the shape function, the vector $\mathbf{a}(\xi)$ is analogous to the nodal values same as in FEM. The radial function $a_{j}(\xi)$ represents the variation of the scattered wave potential in the radial axis $\xi$ at each node $j$, and the shape function $\mathbf{N}(s)$ interpolates between the nodal potential values in the circumferential axis $s$.

By performing scaled boundary transformation, the operator $\nabla$ can be expressed 
160 as [9]:

$$
\nabla=\mathbf{b}_{1}(s) \frac{\partial}{\partial \xi}+\frac{1}{\xi} \mathbf{b}_{2}(s) \frac{\partial}{\partial s},
$$

161 where $\mathbf{b}_{1}(s)$ and $\mathbf{b}_{2}(s)$ are dependent only on the boundary definition

$$
\mathbf{b}_{1}(s)=\frac{1}{|J|}\left\{\begin{array}{c}
y_{s}(s)_{, s} \\
-x_{s}(s)_{, s}
\end{array}\right\}, \quad \mathbf{b}_{2}(s)=\frac{1}{|J|}\left\{\begin{array}{c}
-y_{s}(s) \\
x_{s}(s)
\end{array}\right\},
$$

162 and $|J|$ is the Jacobian at the boundary

$$
|J|=x_{s}(s) y_{s}(s)_{, s}-y_{s}(s) x_{s}(s)_{, s} .
$$

163 From Eqs. (15) and (22), the approximate velocity can be expressed as

$$
v_{A}(\xi, s)=\mathbf{B}_{1}(s) \mathbf{a}(\xi)_{, \xi}+\frac{1}{\xi} \mathbf{B}_{2}(s) \mathbf{a}(\xi)
$$

164 where

$$
\mathbf{B}_{1}(s)=\mathbf{b}_{1}(s) \mathbf{N}(s), \quad \mathbf{B}_{2}(s)=\mathbf{b}_{2}(s) \mathbf{N}(s)_{, s} .
$$

165 Applying the Galerkin approach, the weighting function $w$ can be formulated using 166 the same shape function as in Eq. (21)

$$
w(\xi, s)=\mathbf{N}(s) \mathbf{w}(\xi)=\mathbf{w}(\xi)^{T} \mathbf{N}(s)^{T}
$$

167 Substituting Eqs. (21), (22), (26) and (27) into Eq. (19) results in 


$$
\begin{aligned}
& \int_{\Omega}\left[\mathbf{B}_{1}(s) \mathbf{w}(\xi)_{, \xi}+\frac{1}{\xi} \mathbf{B}_{2}(s) \mathbf{w}(\xi)\right]^{T}\left[\mathbf{B}_{1}(s) \mathbf{a}(\xi)_{, \xi}+\frac{1}{\xi} \mathbf{B}_{2}(s) \mathbf{a}(\xi)\right] d \Omega \\
& -\int_{\Omega} k^{2} \mathbf{w}(\xi)^{T} \mathbf{N}(s)^{T} \mathbf{N}(s) \mathbf{a}(\xi) d \Omega-\oint_{\Gamma} \mathbf{w}(\xi)^{T} \mathbf{N}(s)^{T} \bar{v}_{n} d \Gamma=0,
\end{aligned}
$$

where the incremental volume is [9]

$$
d \Omega=|J| \xi d \xi d s
$$

169 For convenience, coefficient matrices are introduced here as

$$
\begin{aligned}
\mathbf{E}_{0} & =\int_{S} \mathbf{B}_{1}(s)^{T} \mathbf{B}_{1}(s)|J| d s \\
\mathbf{E}_{1} & =\int_{S} \mathbf{B}_{2}(s)^{T} \mathbf{B}_{1}(s)|J| d s \\
\mathbf{E}_{2} & =\int_{S} \mathbf{B}_{2}(s)^{T} \mathbf{B}_{2}(s)|J| d s \\
\mathbf{M}_{0} & =\int_{S} \mathbf{N}(s)^{T} \mathbf{N}(s)|J| d s \\
\mathbf{F}_{s}(\xi) & =\mathbf{N}\left(s_{0}\right)^{T}\left(-\bar{v}_{n}\left(\xi, s_{0}\right)\right)\left|J\left(s_{0}\right)\right|+\mathbf{N}\left(s_{1}\right)^{T}\left(-\bar{v}_{n}\left(\xi, s_{1}\right)\right)\left|J\left(s_{1}\right)\right| .
\end{aligned}
$$

170 The above integrals Eqs. (30)-(33) can be computed element by element and as171 sembled together for the entire boundary. Expanding Eq. (28) and integrating the 172 terms containing $\mathbf{w}(\xi)_{, \xi}$ by parts with respect to $\xi$ using Green's theorem leads to 


$$
\begin{aligned}
& \mathbf{w}\left(\xi_{1}\right)^{T}\left[\mathbf{E}_{0} \xi_{1} \mathbf{a}\left(\xi_{1}\right)_{, \xi}+\mathbf{E}_{1}^{T} \mathbf{a}\left(\xi_{1}\right)-\int_{S} \mathbf{N}(s)^{T}\left(\bar{v}_{n}\left(\xi_{1}, s\right)\right) \xi_{1} d s\right] \\
- & \mathbf{w}\left(\xi_{0}\right)^{T}\left[\mathbf{E}_{0} \xi_{0} \mathbf{a}\left(\xi_{0}\right)_{, \xi}+\mathbf{E}_{1}^{T} \mathbf{a}\left(\xi_{0}\right)+\int_{S} \mathbf{N}(s)^{T}\left(\bar{v}_{n}\left(\xi_{0}, s\right)\right) \xi_{0} d s\right] \\
- & \int_{\xi_{0}}^{\xi_{1}} \mathbf{w}(\xi)^{T}\left[\mathbf{E}_{0} \xi \mathbf{a}(\xi)_{, \xi \xi}+\left(\mathbf{E}_{0}+\mathbf{E}_{1}^{T}-\mathbf{E}_{1}\right) \mathbf{a}(\xi)_{, \xi}-\mathbf{E}_{2} \frac{1}{\xi} \mathbf{a}(\xi)+k^{2} \xi \mathbf{M}_{0} \mathbf{a}(\xi)-\mathbf{F}_{s}(\xi)\right] d \xi \\
& =0 .
\end{aligned}
$$

To satisfy all sets of weighting function $\mathbf{w}(\xi)$, the following conditions must be satisfied:

$$
\begin{gathered}
\mathbf{q}\left(\xi_{1}\right)=\int_{S} \mathbf{N}(s)^{T}\left(\bar{v}_{n}\left(\xi_{1}, s\right)\right) \xi_{1} d s, \\
\mathbf{q}\left(\xi_{0}\right)=-\int_{S} \mathbf{N}(s)^{T}\left(\bar{v}_{n}\left(\xi_{0}, s\right)\right) \xi_{0} d s, \\
\mathbf{E}_{0} \xi^{2} \mathbf{a}(\xi)_{, \xi \xi}+\left(\mathbf{E}_{0}+\mathbf{E}_{1}^{T}-\mathbf{E}_{1}\right) \xi \mathbf{a}(\xi)_{, \xi}-\mathbf{E}_{2} \mathbf{a}(\xi)+k^{2} \xi^{2} \mathbf{M}_{0} \mathbf{a}(\xi)=\xi \mathbf{F}_{s}(\xi),
\end{gathered}
$$

$$
\mathbf{q}(\xi)=\mathbf{E}_{0} \xi \mathbf{a}(\xi)_{, \xi}+\mathbf{E}_{1}^{T} \mathbf{a}(\xi)
$$

Eq. (38) is the so-called scaled boundary finite-element equation. By introducing the shape function, the Helmholtz equation has been weakened in the circumferential direction, so that the governing partial differential equation is transformed to an ordinary matrix differential equation in radial direction. The rank of matrices $\mathbf{E}_{0}$, $\mathbf{E}_{1}, \mathbf{E}_{2}, \mathbf{M}_{0}$ and vector $\mathbf{a}(\xi)$ is $m$ (where $m$ is the number of nodes in the curve $S$ ). In the present study, the side-faces either coincide or are impermeable so that the term $\mathbf{F}_{s}(\xi)$ vanishes. Therefore, the final governing equation, Eq. (38), is a homo- 
geneous second-order ordinary matrix differential equation in terms of matrix of rank $m$.

Boundary conditions, Eqs. (6) and (7) or Eqs. (12) and (10) - (11), are weakened in the form of Eqs. (37) and (36) respectively, indicating the relationship between the integrated nodal flow on the boundary and the velocity potentials of the nodes. For the wave diffraction problem in the unbounded region $S_{0}, \xi_{0}=1$ on the boundary of exterior porous cylinder and $\xi_{1}=+\infty$ at infinity. For the boundary-value problem in the bounded region $S_{j}(j \neq 0), \xi_{0}=0$ and $\xi_{1}=1$.

\subsection{Solution procedure}

\subsubsection{Solution for unbounded sub-domain $S_{0}$}

For the exterior porous circular cylinder, we have

$$
x_{s}(s)=c \cos (s / c), \quad y_{s}(s)=c \sin (s / c) \text {. }
$$

From Eqs. (20), (23), (24), (26) and (30)-(33), $x_{s}(s)_{, s}, y_{s}(s)_{, s}, \mathbf{b}_{1}(s), \mathbf{b}_{2}(s),|J|$, $\mathbf{B}_{1}(s), \mathbf{B}_{2}(s), \mathbf{E}_{0}, \mathbf{E}_{1}, \mathbf{E}_{2}$, and $\mathbf{M}_{0}$ can be calculated accordingly. The following relationships hold:

$$
\begin{array}{r}
\mathbf{E}_{1}=0 \cdot \mathbf{I}, \quad \mathbf{E}_{0}^{-1} \mathbf{M}_{0}=c^{2} \mathbf{I}, \\
\mathbf{E}_{0}=\frac{1}{c} \int_{S} \mathbf{N}(s)^{T} \mathbf{N}(s) d s,
\end{array}
$$

where $\mathbf{I}$ is the identity matrix of rank $m$.

Using Eq. (41), pre-multiplying both sides of Eq. (38) by $\mathbf{E}_{0}^{-1}$ and simplifying, we 
199 have

$$
\zeta^{2} \mathbf{a}(\zeta)_{, \zeta \zeta}+\zeta \mathbf{a}(\zeta)_{, \zeta}-\mathbf{E}_{0}^{-1} \mathbf{E}_{2} \mathbf{a}(\zeta)+\zeta^{2} \mathbf{a}(\zeta)=0
$$

200

where

$$
\zeta=k c \xi
$$

Eq. (43) is the matrix form of Bessel's differential equation. Considering the Sommerfeld radiation condition Eq. (7), it is logical to select $H_{r_{j}}(\zeta) \mathbf{T}_{j}$ as a base solution of Eq. (43) in region $S_{0}$.

The solution for $\mathbf{a}_{0}(\zeta)$ is then expressed in the series form:

$$
\mathbf{a}_{0}^{S}(\zeta)=\sum_{j=1}^{m} c_{j} H_{r_{j}}(\zeta) \mathbf{T}_{j}=\mathbf{T H}(\zeta) \mathbf{C},
$$

where $\mathbf{T}_{j}$ are vectors of rank $m, c_{j}$ are coefficients, $H_{r_{j}}(\zeta)$ are the Hankel functions of the first kind, and

$$
\begin{aligned}
\mathbf{T} & =\left[\mathbf{T}_{1}, \mathbf{T}_{2}, \cdots, \mathbf{T}_{m}\right], \\
\mathbf{C} & =\left[c_{1}, c_{2}, \cdots, c_{m}\right]^{T}, \\
\mathbf{H}(\xi) & =\operatorname{diag}\left[H_{r_{1}}(k c \xi), H_{r_{2}}(k c \xi), \cdots, H_{r_{m}}(k c \xi)\right],
\end{aligned}
$$




$$
\mathbf{q}_{0}^{S}(k c)=k c \mathbf{E}_{0} \mathbf{T} \mathbf{H}_{b h} \mathbf{T}^{-1} \mathbf{a}_{0}^{S}(k c)=-\left[\int_{S} \mathbf{N}(s)^{T} \mathbf{N}(s) d s\right] \overline{\mathbf{v}}_{0 n}^{S},
$$

$$
\sum_{j=1}^{m}\left(\mathbf{E}_{0}^{-1} \mathbf{E}_{2}-r_{j}^{2} \mathbf{I}\right) \mathbf{T}_{j} \cdot c_{j} H_{r_{j}}(\zeta)=0 .
$$

$$
\left(\mathbf{E}_{0}^{-1} \mathbf{E}_{2}-r_{j}^{2} \mathbf{I}\right) \mathbf{T}_{j}=0
$$

$$
\mathbf{q}_{0}^{S}(k c)=\mathbf{E}_{0} k c \sum_{j=1}^{m} c_{j} H_{r_{j}}^{\prime}(k c) \mathbf{T}_{j}=-\left[\int_{S} \mathbf{N}(s)^{T} \mathbf{N}(s) d s\right] \overline{\mathbf{v}}_{0 n}^{S},
$$

${ }_{218}$ where $\overline{\mathbf{v}}_{0 n}^{S}$ is the vector of nodal normal velocity of scattered wave on $\Gamma_{c}$.

219 Using Eq.(45), the boundary condition on $\Gamma_{c}$ can be written as

$$
\begin{aligned}
\zeta^{2} H_{r_{j}}^{\prime \prime}(\zeta) & =-\zeta^{2} H_{r_{j}}(\zeta)+\zeta H_{r_{j}+1}(\zeta)-r_{j} H_{r_{j}}(\zeta)+r_{j}^{2} H_{r_{j}}(\zeta) \\
\zeta H_{r_{j}}^{\prime}(\zeta) & =-\zeta H_{r_{j}+1}(\zeta)+r_{j} H_{r_{j}}(\zeta)
\end{aligned}
$$

where 
50

51

52

$221 \quad$ 2.3.2 Solution for bounded sub-domain $S_{j}(j=1,2, \cdots, q)$

222

Define

$$
\mathbf{H}_{b h}=\operatorname{diag}\left[H_{r_{1}}(k c)^{\prime} / H_{r_{1}}(k c), \cdots, H_{r_{m}}^{\prime}(k c) / H_{r_{m}}(k c)\right] .
$$

$$
\mathbf{X}(\xi)=\left\{\begin{array}{c}
\mathbf{a}(\xi) \\
\mathbf{q}(\xi)
\end{array}\right\}
$$

223 Eq. (38) can be written as

$$
\zeta \mathbf{X}(\zeta)_{, \zeta}=-\mathbf{Z X}(\zeta)-\zeta^{2} \mathbf{M} \mathbf{X}
$$

224 where

$$
\begin{gathered}
\zeta=k a \xi, \\
\mathbf{M}=\frac{1}{a^{2}}\left[\begin{array}{ll}
0 & 0 \\
& \\
\mathbf{M}_{0} & 0
\end{array}\right],
\end{gathered}
$$

$$
\mathbf{Z}=\left[\begin{array}{cc}
\mathbf{E}_{0}^{-1} \mathbf{E}_{1}^{T} & -\mathbf{E}_{0}^{-1} \\
-\mathbf{E}_{2}+\mathbf{E}_{1} \mathbf{E}_{0}^{-1} \mathbf{E}_{1}^{T} & -\mathbf{E}_{1} \mathbf{E}_{0}^{-1}
\end{array}\right]
$$


The eigenvalue problem is formulated as

$$
\mathbf{Z V}=-\mathbf{V} \boldsymbol{\Lambda}
$$

where

$$
\boldsymbol{\Lambda}=\left[\begin{array}{cc}
\boldsymbol{\Lambda}_{0} & 0 \\
0 & -\boldsymbol{\Lambda}_{0}
\end{array}\right]
$$

Usually there is one zero eigenvalue in $\Lambda_{0}$ (marked as $\left.\lambda_{m}=0\right)$, indicating a constant velocity potential component in the solution domain. This behaviour, however, leads to two linearly dependant eigenvectors in $\mathbf{V}\left(\mathbf{V}_{m}\right.$ and $\left.\mathbf{V}_{m+1}\right)$, making the matrix of eigenvectors $\mathbf{V}$ irreversible.

Solving the eigenvalue problem of

$$
\mathbf{Z}^{2} \mathbf{W}=-\mathbf{W} \bar{\Lambda}
$$

and marking the eigenvector corresponding to the zero eigenvalue as $\mathbf{W}_{m}$, a reversible Jordan matrix is constructed as

$$
\mathbf{J}_{j}= \begin{cases}\mathbf{V}_{j} & 1 \leq j<m \quad \text { or } \quad m+1<j \leq 2 m, \\ -\mathbf{Z} \mathbf{W}_{j} & j=m, \\ \mathbf{W}_{j-1} & j=m+1,\end{cases}
$$

with the property of

$$
\mathbf{Z} \mathbf{J}=-\mathbf{J} \hat{\Lambda}
$$


where

233

234

$$
\mathbf{X}(\zeta)=\mathbf{J R}(\zeta) \zeta^{\mathbf{\Lambda}} \zeta^{\mathbf{U}} \mathbf{D}
$$

where $\mathbf{U}$ is an upper-triangular matrix with zeros on the diagonal, $\mathbf{D}$ is a coefficient vector, and

$$
\mathbf{R}(\zeta)=\mathbf{I}+\zeta^{2} \mathbf{R}_{1}+\zeta^{4} \mathbf{R}_{2}+\cdots+\zeta^{2 k} \mathbf{R}_{k}+\cdots
$$

Writing $\mathbf{Y}(\zeta)=\zeta^{\mathbf{U}}$ and $\mathbf{K}(\zeta)=\mathbf{J R}(\zeta)$, and partitioning all the matrices into block matrix with $m \times m$ dimensions and block vector with $m \times 1$ dimensions respectively, Eq. (67) becomes

$$
\mathbf{X}(\zeta)=\left[\begin{array}{ll}
\mathbf{K}_{11} & \mathbf{K}_{12} \\
\mathbf{K}_{21} & \mathbf{K}_{22}
\end{array}\right]\left[\begin{array}{ll}
\zeta^{\mathbf{\Lambda}_{0}} & 0 \\
0 & \zeta^{-\mathbf{\Lambda}_{0}}
\end{array}\right]\left[\begin{array}{ll}
\mathbf{Y}_{11} & \mathbf{Y}_{12} \\
0 & \mathbf{Y}_{22}
\end{array}\right]\left[\begin{array}{l}
\mathbf{D}_{1} \\
\mathbf{D}_{2}
\end{array}\right]
$$

241 The value at $\zeta=0$ should be finite, thus $\mathbf{D}_{2}=0$. 
Define

$$
\begin{aligned}
\mathbf{A}(\zeta) & =\mathbf{K}_{11}(\zeta) \zeta^{\mathbf{\Lambda}_{0}} \mathbf{Y}_{11}(\zeta) \\
\mathbf{Q}(\zeta) & =\mathbf{K}_{21}(\zeta) \zeta^{\mathbf{\Lambda}_{0}} \mathbf{Y}_{11}(\zeta)
\end{aligned}
$$

243

then

$$
\begin{aligned}
& \mathbf{a}(\zeta)=\mathbf{A}(\zeta) \mathbf{D}_{1}, \\
& \mathbf{q}(\zeta)=\mathbf{Q}(\zeta) \mathbf{D}_{1} .
\end{aligned}
$$

$$
\mathbf{q}(\zeta)=\mathbf{Q}(\zeta) \mathbf{A}^{-1}(\zeta) \mathbf{a}(\zeta)=\mathbf{K}_{21}(\zeta) \mathbf{K}_{11}^{-1}(\zeta) \mathbf{a}(\zeta)
$$

Assembling the matrices in all the sub-domains and noting the boundary conditions (6) and (9)-(11), the whole problem can then be solved.

All the other physical properties of engineering interest including velocity, surface elevation, and pressure can now be determined based on the velocity potentials by Eqs. (15)-(17). The total wave force can then be obtained by integrating the pressure along the body boundary of the cylinder.

The following point is worth noting regarding the use of different base solutions, i.e. Hankel function for unbounded sub-domain and power series for bounded subdomains. Similar to the approach of Wolf [9] in obtaining a solution for soilstructure interaction, here a power series is adopted in the form $\left(\sum_{0}^{\infty} \mathbf{C}_{m} \bar{\xi}^{m}\right)$ in bounded sub-domains. The solutions are obtained as series expansions to limited radial distance $(c)$, and the computation has revealed that the solution procedure is very accurate and efficient. However, in the unbounded sub-domain, the solution in the form of algebraic series $\left(\sum_{0}^{\infty} \mathbf{C}_{m} \bar{\xi}^{-m}\right)$ would involve sums to infinity. For large 
values of $\bar{\xi}$ the series approaches the exact solution rapidly and only a few terms

in the series need to be computed. However, this is only the case at the cylinder boundary $(\bar{\xi}=k c)$ for high frequency waves. For low frequency waves, the series hardly converges to the exact solution. The Hankel function, on the other hand, is a perfect choice in unbounded domain to ensure the radiation condition at infinity being satisfied.

\section{Model validation and applications}

In this section, the SBFEM model is first validated by comparing its semi-analytical predictions with analytical solutions and published results using other numerical methods or experiments for special cases such as wave diffraction by a circular cylinder and a square cylinder. Then the model is further applied to more complicated interaction problems between waves and one and two rectangular cylindrical structures with variable wave parameters and structure configurations.

\subsection{Wave diffraction by a circular cylinder $\left(G_{0}=0\right)$}

When the porous effect parameter $G_{0}=0$, the external circular cylinder is impermeable, leading to the limiting case of wave diffraction by a circular cylinder. For this special case, there is only an unbounded solution domain and the problem of wave diffraction by a circular cylinder can be solved explicitly by matching the no-slip condition on the cylinder boundary. Due to the symmetry of the physical problem, only half of the circumference needs to be discretised. Three-noded quadratic elements are used in the circumferential direction as shown in Fig. 3.

Accurate evaluation of the wave run-up and the wave exciting forces are of paramount 
unbounded domain

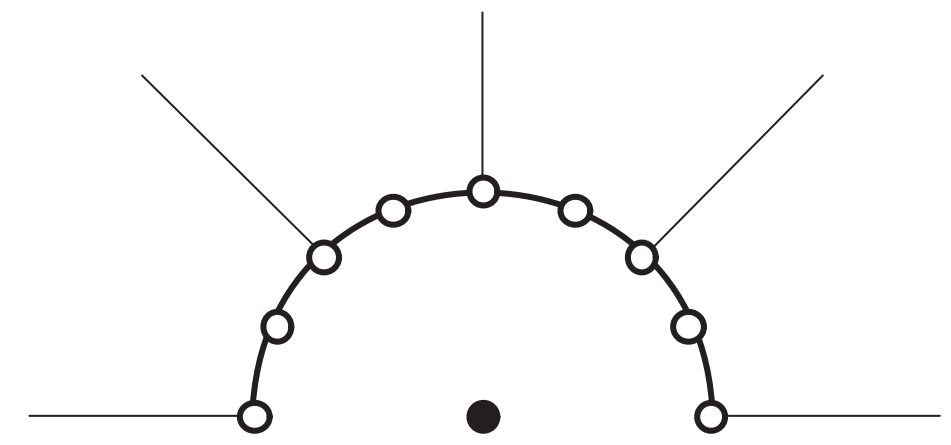

Side face

Scaling centre

Side face

Fig. 3. Scaled boundary finite-element mesh for a circular cylinder

importance in the analysis of dynamic responses of an offshore structure. Fig. 4 is a comparison of wave run-ups on a circular cylinder between the present SBFEM results and the analytical solutions given in [12]. As shown in Fig. 4, for small $k a$ $(=0.5)$, i.e., in the range proposed by [12] where the theory and the experiments have good agreement, the SBFEM results given by even two elements agree well with the analytical solutions. As $k a$ increases from 0.5 to 5.0, the convergence of the SBFEM scheme is clearly evident as the number of elements is increased. Even at $k a=5.0$, accurate numerical results were obtained when merely 8 elements were used for the SBFEM computation.

Fig. 5 is a comparison of wave run-ups computed by the SBFEM, BEM and the analytical solutions of [12] for $k a=2.0$. Forty (40) constant boundary elements are used in BEM. As can be seen in Fig. 5, the SBFEM results obtained with only 4 elements are almost identical to the analytical solutions, a clear demonstration of its superior to traditional BEM.

The relationship between $k a$ and the required element number for computation of wave forces on a circular cylinder are shown in Fig. 6. Excellent computational 


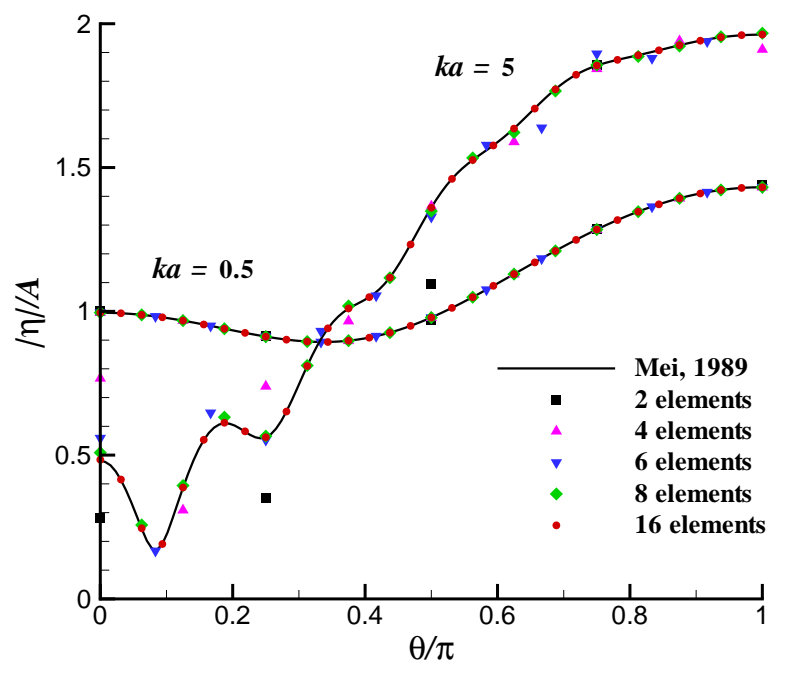

Fig. 4. The run-up of plane wave on a circular cylinder

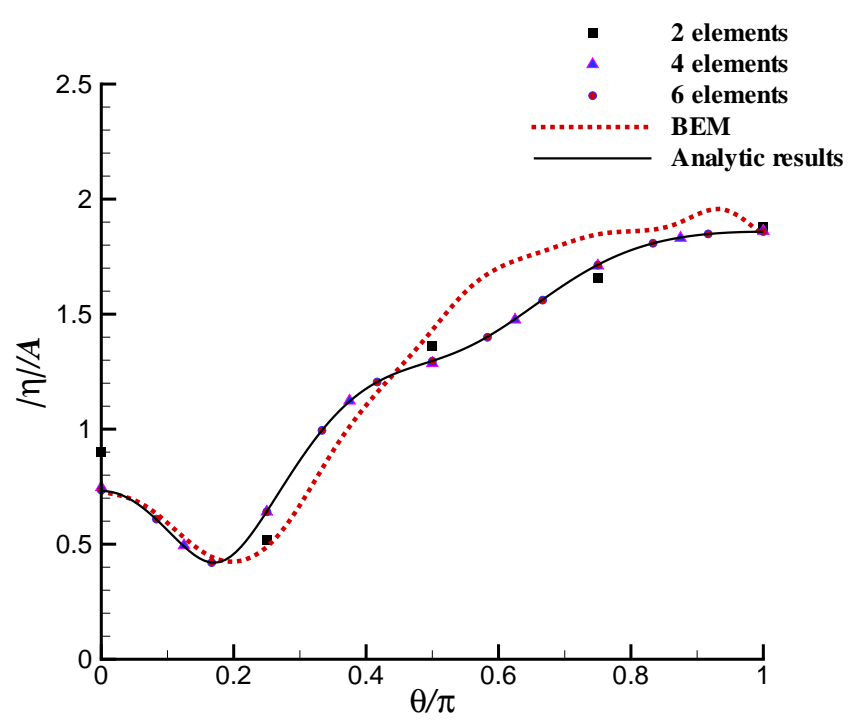

Fig. 5. Wave run-ups on a circular cylinder $(k a=2.0)$.

efficiency and accuracy of the present SBFEM scheme are further demonstrated by examining the hydrodynamic forces. It is seen that the results of the SBFEM model using 8 elements is valid until $k a=10$, and the model returns to satisfactory results in the entire linear range $(0.2<k a<0.65)$ by even using 2 elements. This clearly demonstrates the efficiency of the present SBFEM model. 


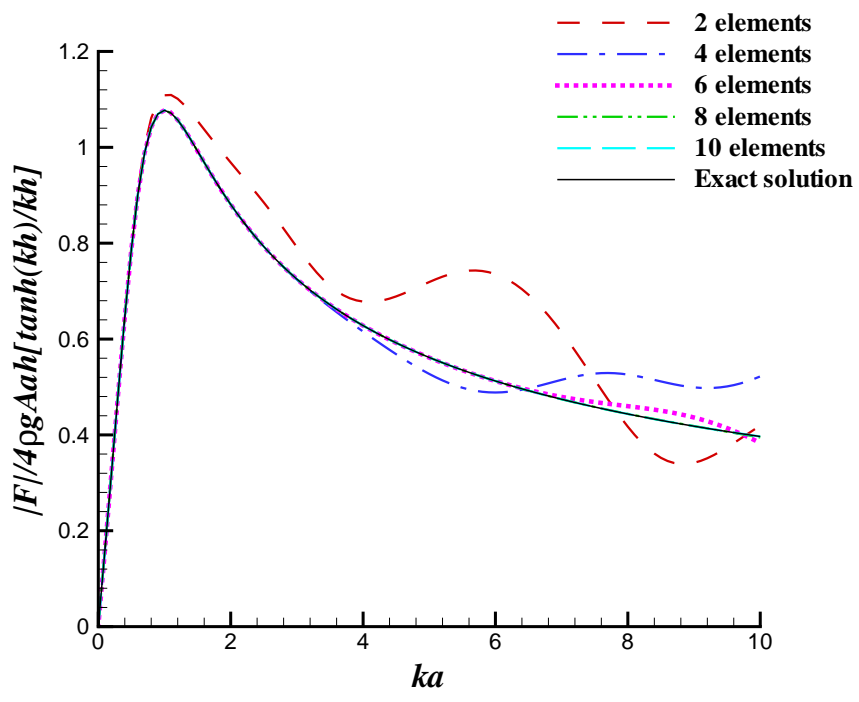

Fig. 6. The relationship between $k a$ and the required elements number for wave forces on a circular cylinder.

3.2 Wave diffraction by a cylindrical structure system other than a circular cylinder

The accuracy and efficiency of the present SBFEM model is demonstrated by the above wave diffraction problem. However, the case of wave interaction with a circular cylinder is relatively simple, the analytical solution exists and accurate numerical results can be easily obtained by traditional numerical methods. As a key element of the present SBFEM model, the introduction of the virtual cylinder to decompose the solution domain into bounded and unbounded sub-domains and apply different strategies in solution techniques in these sub-domains, will be further demonstrated by the following limiting cases. 
circular cylinder, in which no fundamental solutions exist. The solution domain is then divided into one unbounded sub-domain outside the virtual circular cylinder and several bounded sub-domains inside the circular cylinder as shown in Fig. 1. By matching the boundary conditions on the virtual circular cylinder due to $G_{0}=\infty$, the solutions of the bounded and unbounded sub-domains are solved separately.

\subsubsection{Wave diffraction by a single square cylinder}

For a cylinder with sharp corners, the scaling centre in the associated bounded sub-domain is chosen at the corner. The discretisation along the interfaces of the sub-domains with three-node quadratic elements are shown in Fig. 7, where $a$ is the half width of the cylinder in $x$ direction and $b$ is the half length of the cylinder in $y$ direction. For square cylinder, $b=a$. If the physical problem is symmetric (e.g. incident wave angle $\theta=0, \pm \pi / 2, \pi$ ), only half of the sub-domains need to be discretised. In the following validation, $\theta=0$ is chosen so the total elements number are reduced to half and the wave forces in $y$ direction are equal to zero.

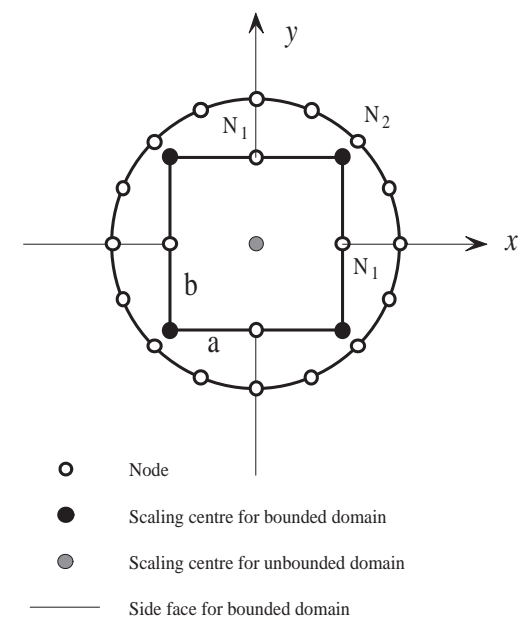

Fig. 7. Scaled boundary finite-element mesh for a square cylinder 


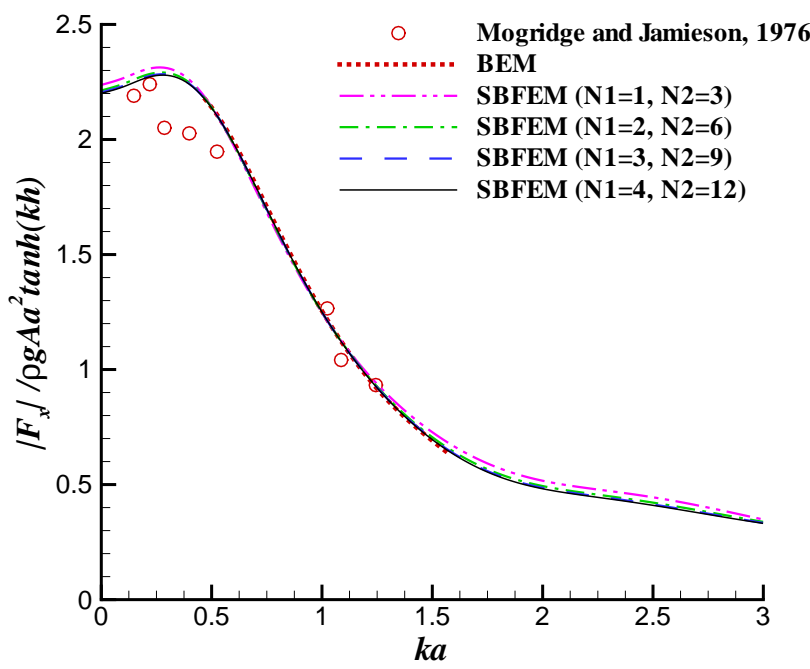

Fig. 8. Comparisons of the nondimensional wave forces on a square cylinder from SBFEM, experiments [13] and BEM.

Mogridge and Jamieson [13] measured the wave forces on a large square caisson. Two square caissons were tested individually in the experiment. One is 12 in. by 12 in., the other is $2 \mathrm{ft}$. by $2 \mathrm{ft}$.. Monochromatic waves were generated in five water depths ranging from 9.7 to 29 in. and nine wave periods were tested from 0.77 to $2.58 \mathrm{~s}$. A number of wave heights were generated for each water depth and period tested [13]. Fig. 8 is a comparison of wave forces computed using the present SBFEM model, experimental data of [13] and BEM solutions. The meshes of the SBFEM solution are shown in Fig. 7, where $N_{1}$ is the element number in one of the interfaces of the interior sub-domains and $N_{2}$ is the element number in one of the interfaces of an interior sub-domain and exterior sub-domain. Convergence test plotted in Fig. 8 shows that the wave forces converge rapidly as the number of elements increases. Even the very coarse mesh $\left(N_{1}=1\right.$ and $\left.N_{2}=3\right)$ achieves excellent results, while the BEM requires finer mesh for the similar accuracy.

Fig. 9 shows the nondimensional wave forces computed using the present SBFEM 


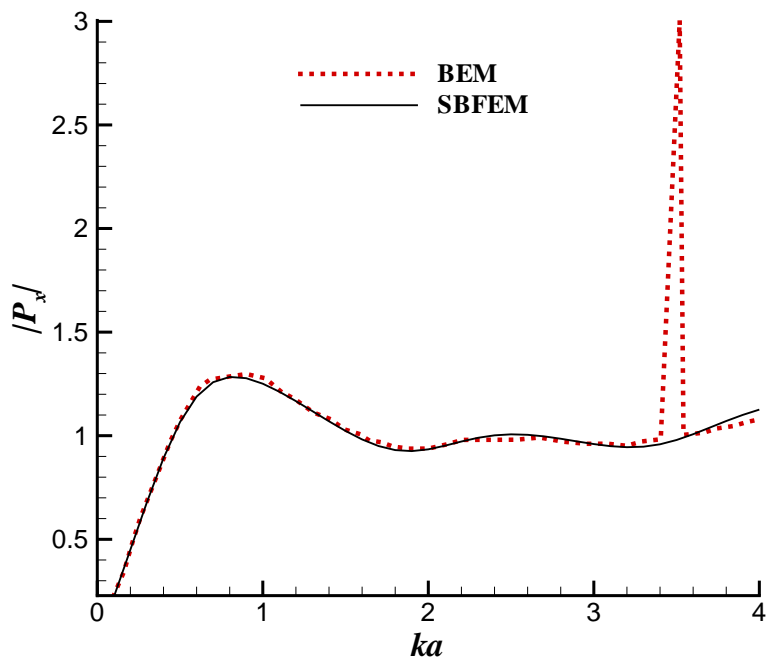

Fig. 9. Comparisons of the nondimensional wave forces on a square cylinder: SBFEM vs BEM.

model and conventional BEM. 16 three-node quadratic elements are used in the BEM solution and a very coarse mesh $\left(N_{1}=1\right.$ and $\left.N_{2}=2\right)$ is chosen in the SBFEM computation. It is seen in Fig. 9 that BEM is unable to provide accurate result around $k a=3.5$ where the irregular frequency occurs. However, the solution given by the present SBFEM model using a very coarse mesh is seen to produce very accurate results without suffering the irregular frequency, a clear demonstration of the superiority of the present SBFEM model.

\subsubsection{Wave diffraction by a rectangular cylinder}

For wave diffraction by a rectangular cylinder, the solution process is very similar to the process in Section 3.2.1. However, as the side lengths are no longer equal ( $b \neq a$ in Fig. 7), the maximum wave forces is different from the forces on the square cylinder. Fig. 10 shows the effect of incident wave angle $\theta$ on the nondimensional total wave forces $\left|F_{T}\right| / 4 \rho g A a^{2} \tanh (k h)$. It is seen in the figure that the maximum wave force on a rectangular cylinder occurs when the incident wave is 
normal to the longer side $\left(\theta=0^{\circ}\right.$ or $\left.90^{\circ}\right)$, while the maximum wave force on a square cylinder always occurs as the incident wave is parallel to the diagonal of the square $\left(\theta=45^{\circ}\right)$. The minimum wave force, however, is not only dependent on the incident wave direction, but also related to the nondimensional incident wave number $k a$. For small $k a$, the minimum wave force arises as the incident wave is normal to the shorter side, while for large $k a$, it appears that minimum force can be resulted from two varying incident angles depending on the configuration and incident wave number. Thus, detailed calculation should be carried out before design to fully optimise the structure configuration according to the dominant incoming wave direction.

The effect of the nondimensional side lengths $k a$ and $k b$ on the nondimensional wave forces $\left|F_{T}\right| / 4 \rho g A b^{2} \tanh (k h)$ and $\left|F_{T}\right| / 4 \rho g A a^{2} \tanh (k h)$ for $\theta=0$ are examined in Figs. 11 and 12 respectively. As $k b$ is the nondimensional length of the side normal to the incident wave, a similar trend of increasing wave forces with different slope as $k b$ increases for all different $k a$ values is observed (Fig. 12). However, as can be seen in Fig. 11, increasing $k a$ (incoming wave direction) may increase or reduce the wave forces on the cylinder depending on the value of $k b$.

\subsection{Wave diffraction by two adjacent rectangular cylinders}

For water wave diffraction by multiple bodies as sketched in Fig. 13, the interaction of the scattered waves of the multiple structures is not negligible. In this section, a cylindrical structure system consists of two rectangular cylinders placed close to each other in a wave field is computed using the present SBFEM model. The results of the nondimensional wave forces $|P|\left(|P|=\left|F_{x}\right| / 4 \rho g \operatorname{Aah}[\tanh (k h) / k h]\right)$ on the two cylinders are compared with BEM solutions for different configurations $d / L$ 


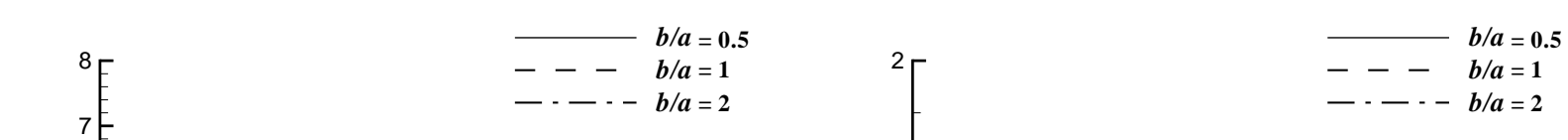

$k a=0.5$
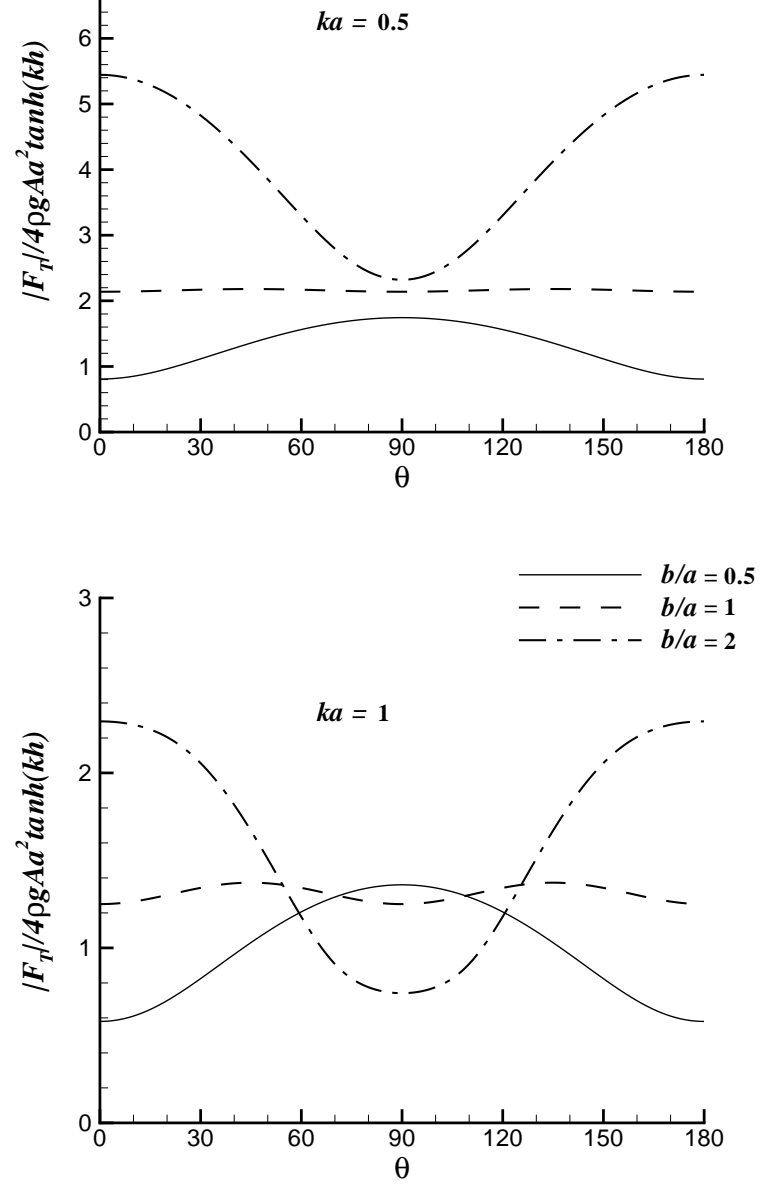

$k a=1.5$
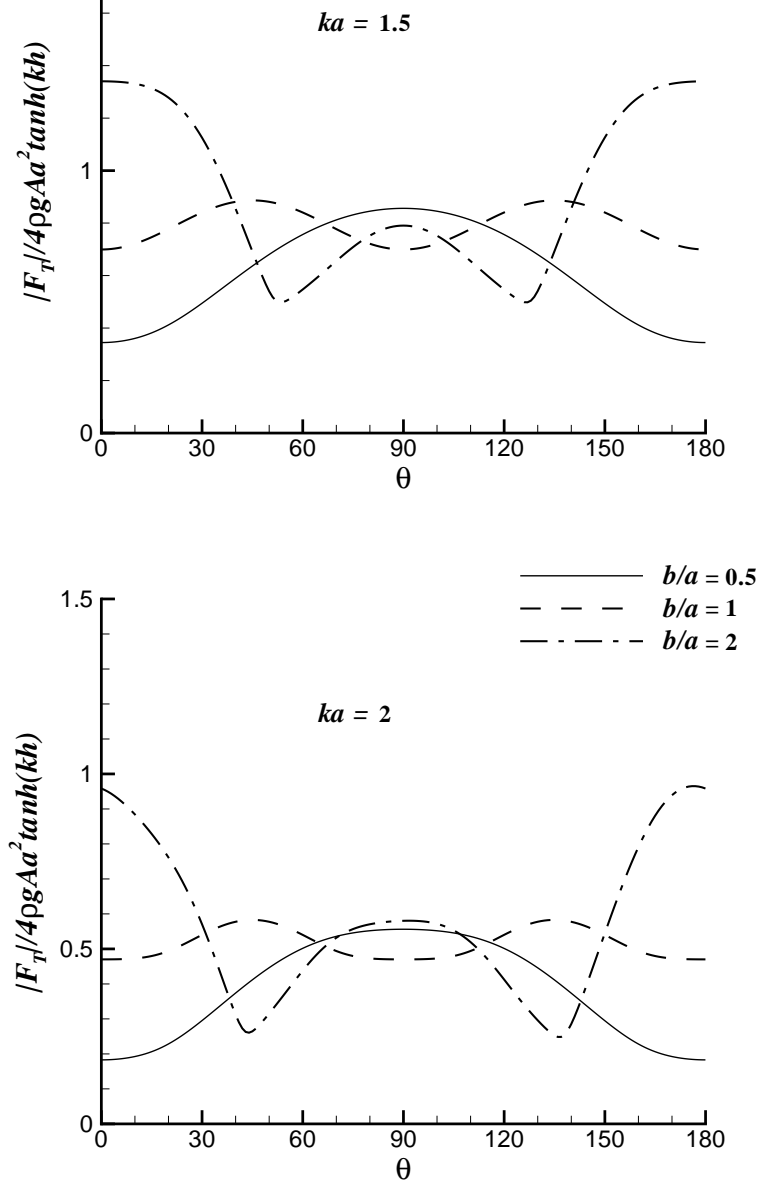

Fig. 10. Variation of nondimensional wave forces on the rectangular cylinder vs. incident wave angle $\theta$.

( $L=2 a, B=2 b) . P_{L}$ and $P_{R}$ represent wave force on left cylinder (upstream) and right cylinder (downstream) respectively. As can be seen in Fig. 14, excellent agreement is achieved between the SBFEM solutions and BEM results for cases of relative spacing between the two cylinders from $d / L=0.1$ to 0.5 . However, it is worth pointing out that the SBFEM computation is based on merely total 14 elements discretised along the cylinder boundaries and interfaces.

Fig. 15 is a plot of wave forces on two cylinders against relative spacing. Wave forces on both cylinders is seen to have a brief increase with increasing spacing 


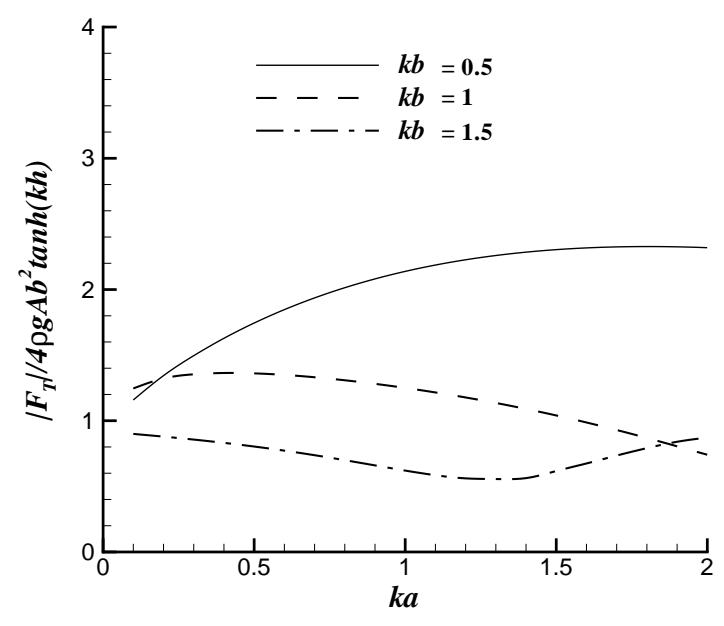

Fig. 11. Variation of nondimensional wave forces $\left|F_{T}\right| / 4 \rho g A b^{2} \tanh (k h)$ on the rectangular cylinder $v s$. side length $k a$.

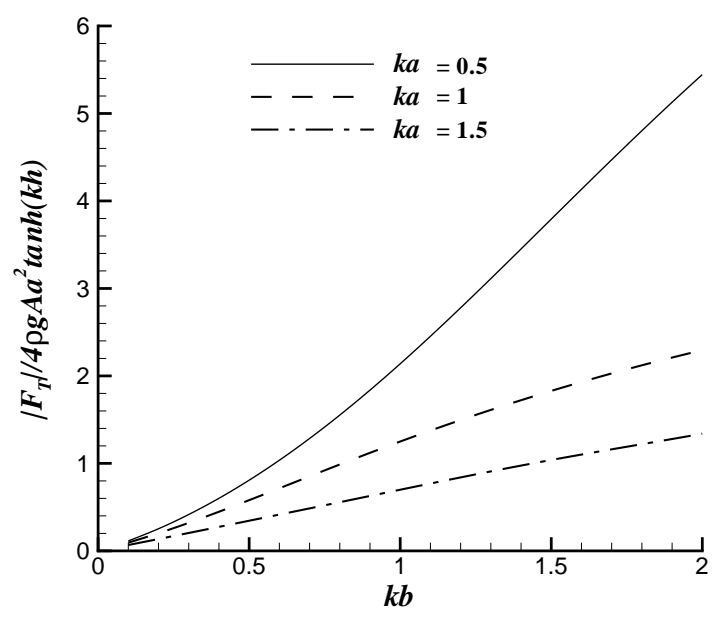

Fig. 12. Variation of nondimensional wave forces $\left|F_{T}\right| / 4 \rho g A a^{2} \tanh (k h)$ on the rectangular cylinder $v s$. side length $k b$.

between the cylidners at very small gap. Such a brief increase is followed by a steady decrease in wave forces on both cylinders as the spacing increases until reaches their respectively minimum at approximately $d / L=1.0$ for this particular incident wave $(k L=2)$. Beyond this value, wave forces on both cylinders tend to increase again as $d / L$ continue to increase. It is interesting to note that for the 
given incident wave, the upstream cylinder tends to experience larger wave force than downstream one when the spacing is small. However, within a range of the spacing close to the side length of the rectangle cross section $(L)$, the wave force on the downstream cylinder appears to be slightly larger than its upstream counterpart. When the spacing continue to increase, the wave force on the upstream cylinder increases in a more rapid path and tends to be greater than that experienced by the cylinder in the downstream.

Fig. 16 shows the influence of the gap between the two cylinders on the wave forces on the two cylinders respectively for given wave conditions $(k L=0.1,0.5,1.0,1.5)$. In general, the upstream cylinder appears to experience larger wave force than the cylinder placed in the downstream. The oscillatory behavour of the wave forces, dependent on the gap, experienced by the two cylinders are the clear evidence of the impact on the hydrodynamics due to the existence of one cylinder to another. By identifying the peak values of the wave forces associated with each configuration, this important characteristic in the forces can be effectively applied in a design to reduce the wave impact on coastal and offshore structures.

Similar to the wave diffraction by a single rectangular cylinder, Fig. 17(a) shows an initial decrease of wave force on the upstream cylinder at low $k L$, followed by a sharp increase as $k L$ increases. For the configuration calculated with relative spacing $d / L=0.25$ to 1.5 , however, the cylinder in the down stream experiences increasing wave force as $k L$ increases even at very small low $k L$ (Fig. 17(b)). Since the flow region in the wake immediate downstream of the left cylinder has been significant altered due the its existence, the cylinder in the downstream interact with a flow field different with the incident wave from far field resulting different hydrodynamic behaviour. Increasing the gap further between the two cylinders, however, the hydrodynamics of the downstream cylinder should reassemble its up- 
stream counterpart as the interference between the two cylinders becomes very weak. It is seen in Fig. 17 that wave forces on both cylinders reach their respective maximums at $k L=1.0 \sim 2.0$ depending on the gap, then rapidly decrease as $k L$ continue to increase.

It is worth noting that the SBFEM model can be applied to more general complex structure system by assigning realistic porous effect parameter $G_{0}$. Example applications in coastal and offshore engineering include rock-filled porous breakwaters outside harbors, and porous outer protective structures with the main structures in its interior, such as the Ekofisk gravity offshore structure in the North Sea.
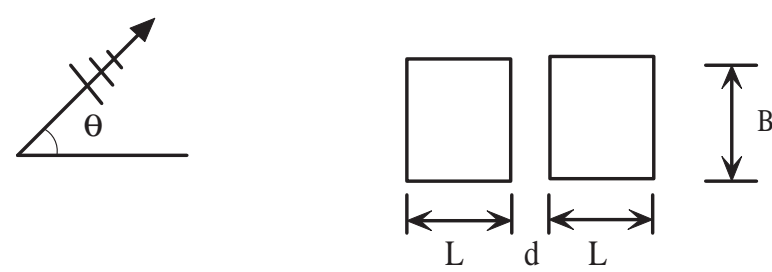

Fig. 13. The sketch of wave diffraction by a cylindrical structure system of twin cylinders

\section{Conclusions}

A new semi-analytical scaled boundary FEM model is developed to simulate the interaction of linear waves with cylindrical structures of arbitrary cross-sectional shapes. Several techniques are applied to ensure that the new SBFEM model is capable of solving wave interaction with single or multiple structures of complex configuration while achieving overall high efficiency and accuracy. The solution domain is partitioned by the introduction of a virtual porous circular cylinder surrounding the structures. A set of the boundary-value problems in an unbounded sub-domain and several bounded sub-domains are then solved semi-analytically by using different base solutions. Computations of different cases of complex config- 

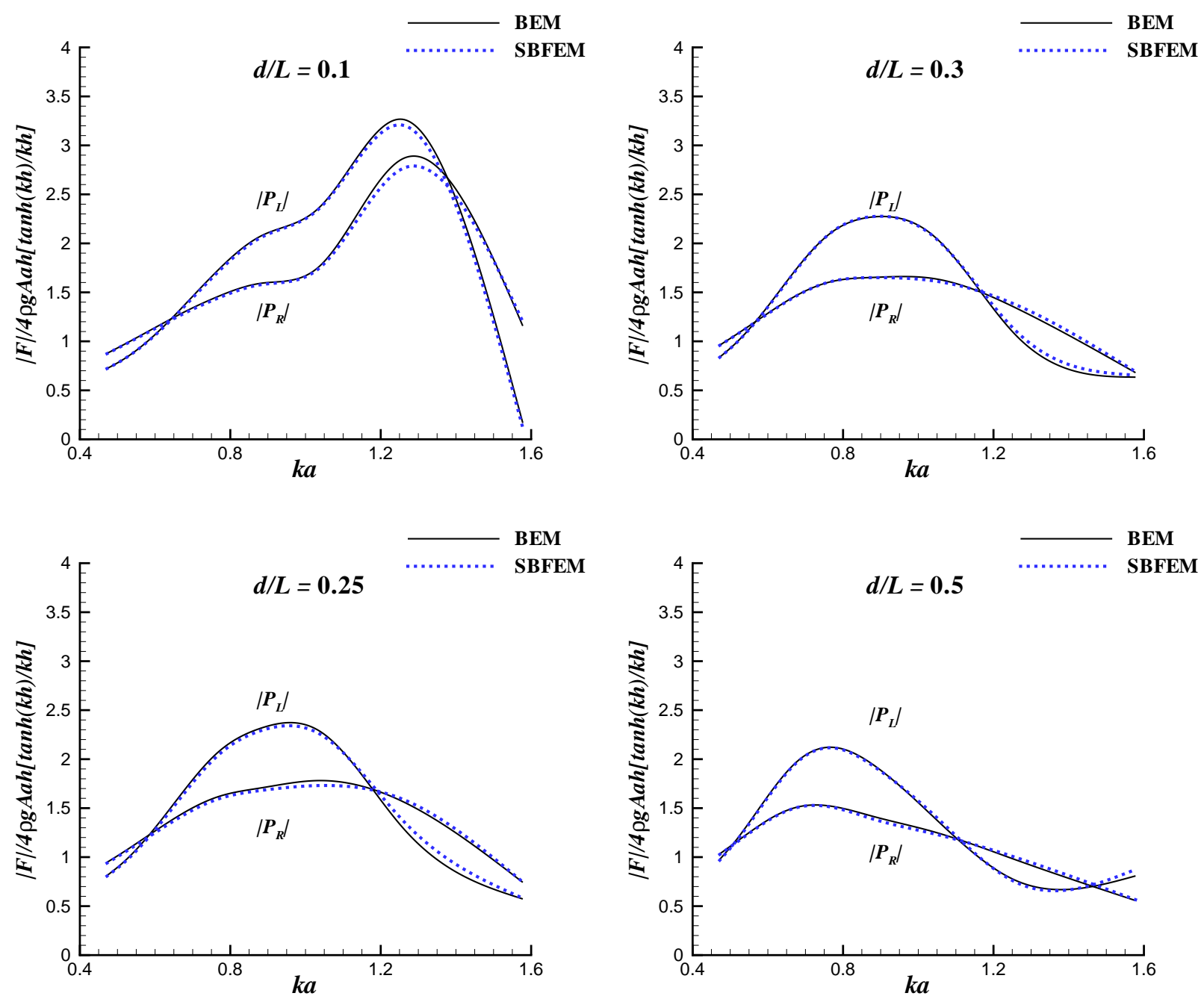

Fig. 14. Comparison of the nondimensional wave forces $\left|F_{x}\right| / 4 \rho g A a h[\tanh (k h) / k h]$ on the twin cylinders $v s . k a$.

uration have demonstrated significant advantages exhibited in the present SBFEM

model including a reduction of one in the spatial dimension is achieved with the solution procedure as the governing equations are solved analytically in the radial direction; the new technique requires no help from any fundamental solutions as required by conventional boundary element method; choice of the base solution in the form of Hankel function of the first kind for the unbounded sub-domain while applying the power series for the bounded sub-domains to further improve the com- 

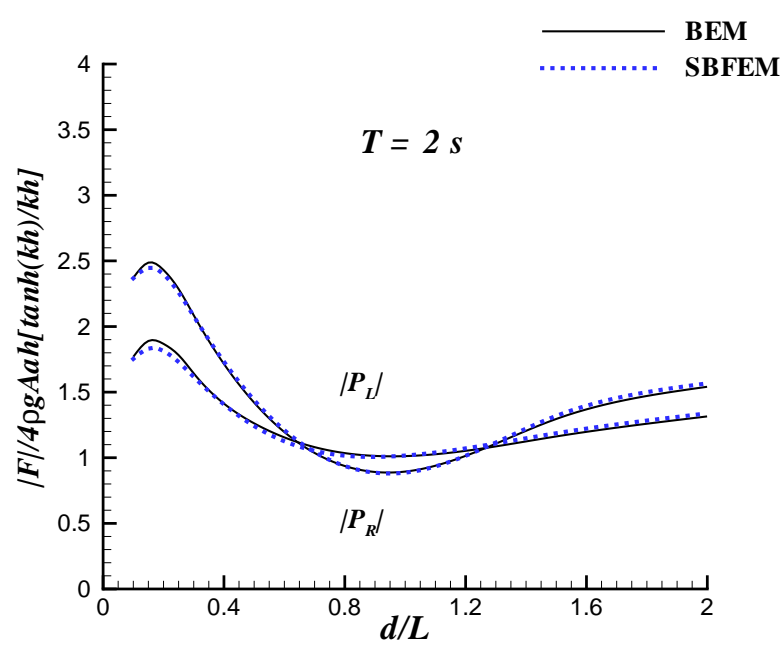

Fig. 15. Comparison of the nondimensional wave forces $\left|F_{x}\right| / 4 \rho g A a h[\tanh (k h) / k h]$ on the twin cylinders $v s$. distance $d / L(T=2 s)$.
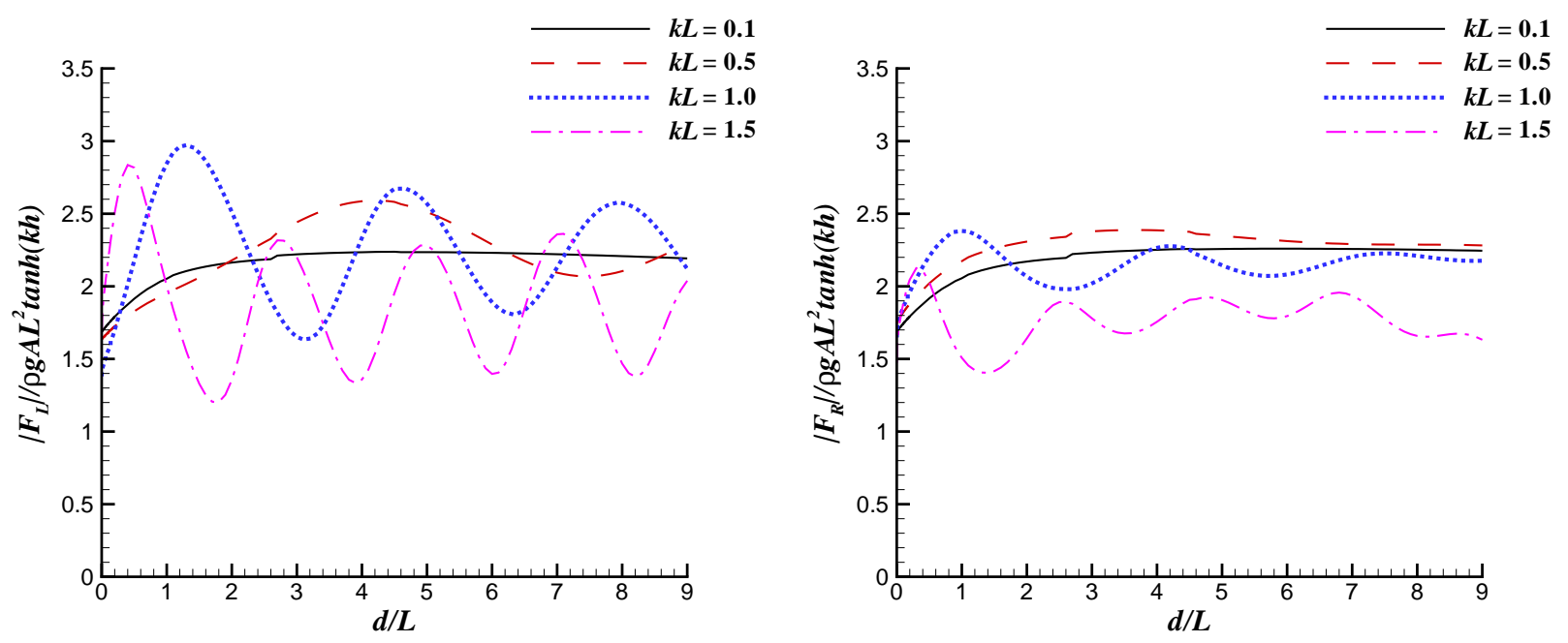

Fig. 16. Variation of nondimensional wave forces $\left|F_{x}\right| / \rho g A L^{2} \tanh (k h)$ on the twin cylinders $v s$. distance $d / L$.

putational accuracy and efficiency.

The newly developed semi-analytical method is shown to reproduce the analytical

solutions and other published results for all the physical properties including wave

run-ups and wave forces very accurately for wave interaction with simple struc- 

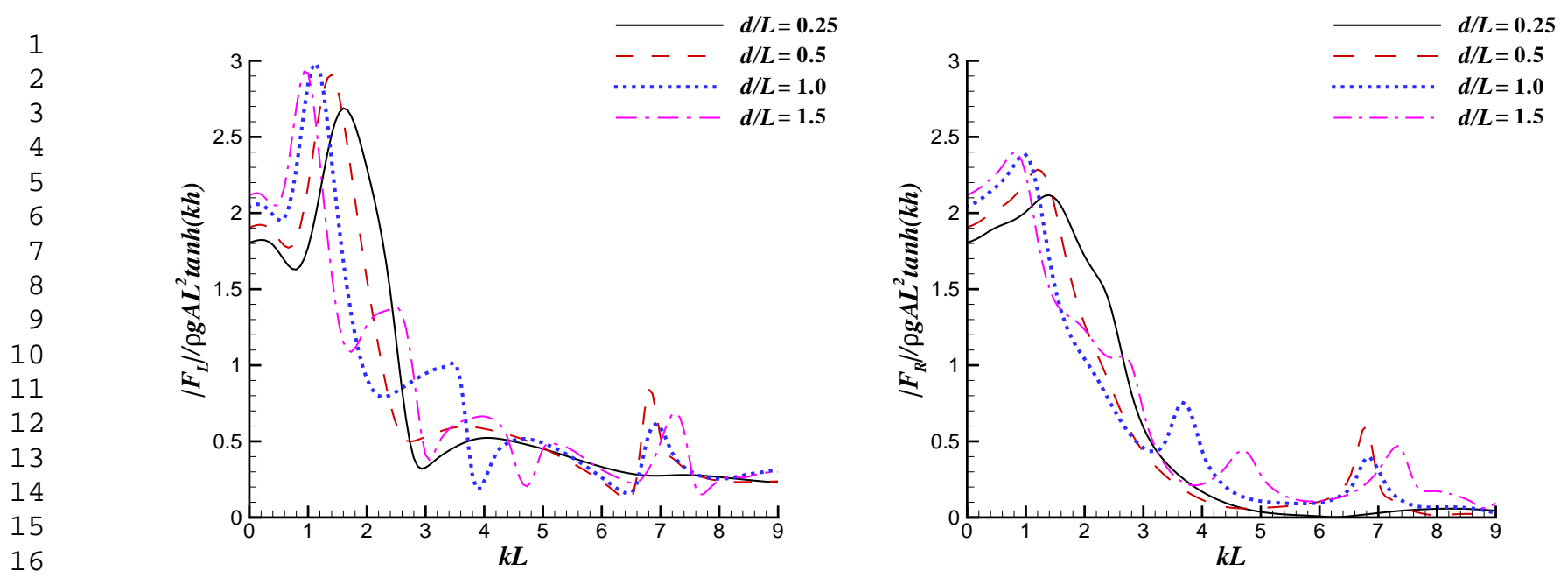

tures. Furthermore, in solving the linear wave interaction with multiple complex structures, the SBFEM model is seen to provide solutions with excellent accuracy at very low computational cost. The method holds promise in solving more practical ocean engineering problems with increased complexity.

Fig. 17. Variation of nondimensional wave forces $\left|F_{x}\right| / \rho g A L^{2} \tanh (k h)$ on the twin cylinders $v s$. side length $k L$ at incident wave angle $\theta=0$.

\section{Acknowledgment}

The first two authors would like to express our gratitude to our co-author, Dr. Subrata Chakrabarti, who has passed away early 2009, for his contribution and dedication throughout the project. The first author is grateful for the postdoctoral fellow- 
[1] Havelock, T.H., 1940, The pressure of water waves upon a fixed obstable, Proc. R. Soc. Lond. A, Vol. 175, pp.409-421.

[2] R.C. MacCamy, R.A. Fuchs, Wave forces on piles: a diffraction theory, Tech. Memo. No. 69, U.S. Army Board, U.S. Army Corp. of Eng. 1954.

[3] S.K. Chakrabarti, W.A. Tam, Interaction of waves with large vertical cylinder, J. Ship Res. 19 (1) (1975) 23-33.

[4] Chen, H.S. and Mei, C.C., 1973, Wave forces on a stationary platform of elliptical shape, J. Ship Res., Vol. 17, No. 2, pp.61-71.

[5] Williams, A.N., 1985, Wave forces on an elliptic cylinder, J. Waterway, Port, Coastal and Ocean Engineering, Vol. 111, No. 2, pp.433-449.

[6] N.J. Shankar, T. Balendra, C.E. Soon, Wave loads on large vertical cylinders: A design method, Ocean Eng. 11 (1) (1984) 65-85.

[7] M.C. Au, C.A. Brebbia, Diffraction of water waves for vertical cylinders using boundary elements, Appl. Math. Model. 7 (2) (1983) 106-114.

[8] D. Lesnic, L. Elliott, D.B. Ingham, Boundary element methods for determining the fluid velocity in potential flow, Eng. anal. bound. elem. 11 (3) (1993) 203-213.

[9] J.P. Wolf, The scaled boundary finite element method, John Wiley \& Sons Ltd, Chichester, England, 2003.

[10] Tao, L., Song, H. and Chakrabarti, S., 2007, Scaled boundary FEM solution of shortcrested wave diffraction by a vertical cylinder, Comput. Method Appl. M., Vol. 197, No. 1-4, pp.232-242.

[11] Chwang, A.T. (1983), A porous wavemaker theory, J. Fluid Mech., 132, 395-406. 
[12] C.C. Mei, The applied dynamics of ocean surface waves, World Scientific, Singapore, 1989.

485

[13] Mogridge, G.R. and Jamieson, W.W., 1976, Wave forces on square caissons, Proc. of 15th Coastal Engineering Conference, Hawaii, USA, pp. 2271-2289. 